\title{
Influence of edges on the exchange bias properties of ferromagnetic/ antiferromagnetic nanodots
}

\author{
V. Baltz, ${ }^{\text {a) }}$ G. Gaudin, P. Somani, and B. Dieny \\ SPINTEC, UMR(8191) CEA/CNRS/UJF/Grenoble INP, INAC, 17 rue des Martyrs, \\ 38054 Grenoble Cedex, France
}

(Received 23 March 2010; accepted 18 May 2010; published online 28 June 2010)

\begin{abstract}
For ferromagnetic (F)/antiferromagnetic (AF) nanodots contributions of AF spins to exchange bias is discussed. The relative weights of AF entities located at the dot edges, at the F/AF interface and in the AF grains were obtained by comparing the blocking temperature distribution of an array of nanodots with that of corresponding continuous film. Due to grain cutting, the grain size distribution is altered. We show here that the dot edges constitute additional locations for the formation of spin-glasslike AF regions at the F/AF interface. The result of patterning is thus twofold and weakens the dot ability to resist thermally activated magnetization reversal. (C) 2010 American Institute of Physics. [doi:10.1063/1.3449123]
\end{abstract}

The ability to pin the magnetization of a ferromagnetic layer in a fixed direction in order to define a reference direction for the spin of conduction electrons is a prerequisite to most spintronic devices. ${ }^{1}$ Exchange bias (EB), which refers to the exchange coupling between a ferromagnet $(\mathrm{F})$ and an antiferromagnet (AF), is most often used for that particular purpose of shifting the hysteresis loop along the magnetic field axis. ${ }^{2}$ Once patterned into arrays of cells, for example in magnetic random access memories, ${ }^{3}$ detrimental distributions of the magnetic properties from cell to cell appear. These distributions mostly concern the coercive field, the EB field $\left(\mathrm{H}_{\mathrm{E}}\right)$ and the blocking temperature $\left(\mathrm{T}_{\mathrm{B}}\right)$ (Refs. 4-6) [the temperature $(\mathrm{T})$ over which AF grains are no longer stable when cycling the $F$ magnetization]. In addition, significant decreases in $\mathrm{H}_{\mathrm{E}}$ and $\mathrm{T}_{\mathrm{B}}$ are often observed upon reducing the lateral cell size below sub-200 $\mathrm{nm}$ dimensions, with the risk of loosing the ability to maintain a reference spin direction for very small memory cells. ${ }^{7}$ Industrial qualification of EB spintronic devices requires the reduction in the width of the distributions $\left(\mathrm{H}_{\mathrm{E}}\right.$ and $\left.\mathrm{T}_{\mathrm{B}}\right)$, the preservation of $\mathrm{H}_{\mathrm{E}}$ larger than the coercive field, and maintaining a significant part of the distribution of $\mathrm{T}_{\mathrm{B}}$ much above working $\mathrm{T}$ despite patterning. The first step toward the fulfillment of these requirements lies in the understanding of the underlying physical origin(s) of the above mentioned effects. For continuous films of F/AF bilayers, we have recently evidenced bimodal distributions of $\mathrm{T}_{\mathrm{B}}{ }^{8}{ }^{8}$ The $\mathrm{T}_{\mathrm{B}}$ distributions consist of two parts: (i) a commonly observed high $\mathrm{T}$ peak associated to thermally activated reversal of the $\mathrm{AF}$ grains spin-lattice ${ }^{6,9,10}$ and (ii) a more unconventional low $\mathrm{T}$ peak ${ }^{8,11}$ ascribed to F/AF interfacial spin-glasslike regions characterized by low freezing $\mathrm{T}^{12,13}$

In the present paper, we focused on the origin of EB and $\mathrm{T}_{\mathrm{B}}$ distributions in nanodots. We evidenced twofold consequences due to patterning which are of importance when considering the down-size scalability of spintronic devices. We identified additional contributions to the $T_{B}$ distribution in EB nanodots with respect to continuous film and understood their origins in terms of edge influence. We were then able to quantify the relative influences of dot edges, F/AF

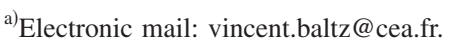

interface, and bulk AF in the EB characteristics.

A sheet film with compositions (from bottom to top) Ta $(3 \mathrm{~nm}) / \mathrm{Cu}(3 \mathrm{~nm}) / \mathrm{Co}(3 \mathrm{~nm}) / \mathrm{IrMn}(7 \mathrm{~nm}) / \mathrm{Pt}(2 \mathrm{~nm})$ was fabricated onto thermally oxidized silicon substrates. Ta $(3 \mathrm{~nm}) / \mathrm{Cu}(3 \mathrm{~nm})$ serve as buffer layers, Co $(3 \mathrm{~nm}) / \mathrm{IrMn}$ $(7 \mathrm{~nm})$ is the F/AF bilayer and $\mathrm{Pt}(2 \mathrm{~nm})$ is a capping layer. IrMn was deposited from an $\mathrm{Ir}_{20} \mathrm{Mn}_{80}$ target. Deposition was obtained at room $\mathrm{T}$ by dc-magnetron sputtering with an $\mathrm{Ar}$ plasma under a pressure of $2.5 \times 10^{-3}$ mbar. Deposition rates ranging between 0.05 and $0.1 \mathrm{~nm} \mathrm{~s}^{-1}$ were used. After deposition, the sheet film was cut into two pieces, one of them being our continuous film. The other part was patterned by a conventional method, which consists in electron beam lithography of an array of holes in a resist, evaporation of a hard mask of Ti $(30 \mathrm{~nm})$, lift-off and ion beam etching. This way, we obtained a $5 \times 5 \mathrm{~mm}^{2}$ array of $180 \times 100 \mathrm{~nm}^{2} \mathrm{mag}$ netic dots with no remaining magnetic materials in between. Spacing between dots of 400 and $200 \mathrm{~nm}$ along the longitudinal and transversal directions, respectively, ensure good magnetostatic decoupling between dots. Scanning electron microscopy (SEM) images of representative parts of the array are shown in Fig. 1 and allow appreciation of the dimensions and shape of the patterned dots.

For both the continuous film and the array of dots, the initial EB was set in situ in the inset of a superconducting quantum interference device (SQUID) magnetometer by postannealing and field-cooling (FC). Initial FC was performed from 450 down to $4 \mathrm{~K}$ with a positive magnetic field, $\mathrm{H}_{\mathrm{FC}}$ of $10 \mathrm{kOe}$, i.e., large enough to saturate the magnetiza-

(a)

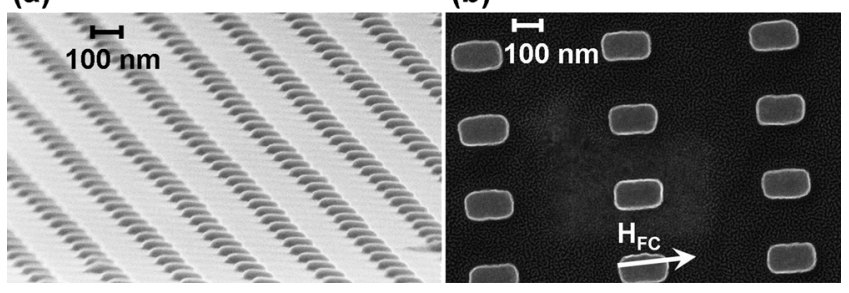

FIG. 1. SEM images of an array of Ta $(3 \mathrm{~nm}) / \mathrm{Cu}(3 \mathrm{~nm}) / \mathrm{Co}(3 \mathrm{~nm}) / \mathrm{IrMn}(7$ $\mathrm{nm}) / \mathrm{Pt}(2 \mathrm{~nm}) 180 \times 100 \mathrm{~nm}^{2}$ dots on a $\mathrm{Si} / \mathrm{SiO}_{2}$ substrate. The images are performed at (a) $45^{\circ}$ and (b) vertical incidence with respect to the sample plane. In (b), the direction of the magnetic field $\left(\mathrm{H}_{\mathrm{FC}}\right)$ during $\mathrm{FC}$ is indicated. 
(a)
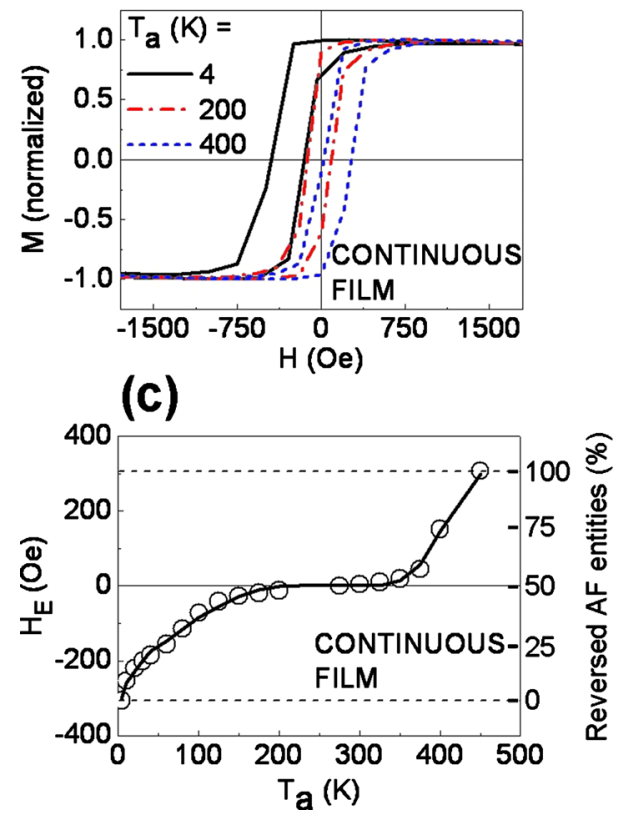

(b)
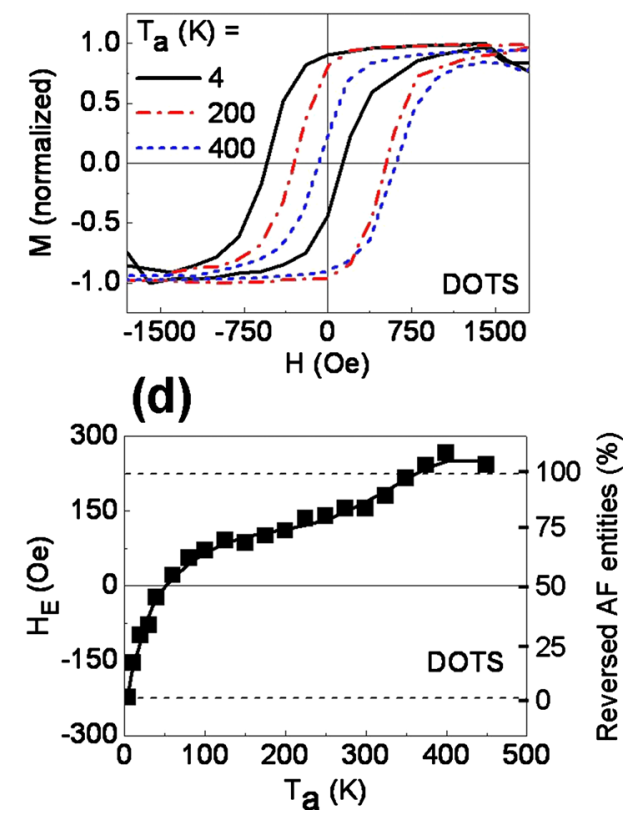

FIG. 2. (Color online) Hysteresis loops measured by SQUID at $4 \mathrm{~K}$ along the FC direction, for different annealing temperatures $\left(\mathrm{T}_{\mathrm{a}}\right)$, for (a) a continuous film and (b) an array of $180 \times 100 \mathrm{~nm}^{2}$ dots. The loops are subsequent to specific cooling procedure. The samples were initially field cooled under a positive saturating field of $10 \mathrm{kOe}$ from 450 down to $4 \mathrm{~K}$. T was then raised up to $\mathrm{T}_{\mathrm{a}}$ and the samples were field cooled under $-10 \mathrm{kOe}$ from $\mathrm{T}_{\mathrm{a}}$ down to $4 \mathrm{~K}$. (c) and (d) corresponding dependences of the loop shift $\left(\mathrm{H}_{\mathrm{E}}\right)$ on $\mathrm{T}_{\mathrm{a}}$. The scales on the right hand side correspond to $\left(\left|\mathrm{H}_{\mathrm{E}}\right|-\left|\mathrm{H}_{\mathrm{E}, \min }\right|\right) /\left(\mathrm{H}_{\mathrm{E}, \max }\right.$ $\left.-\left|\mathrm{H}_{\mathrm{E}, \min }\right|\right)$, where the minimum, $\mathrm{H}_{\mathrm{E} \text {,min }}$ and maximum, $\mathrm{H}_{\mathrm{E} \text {, max }}$ values for $\mathrm{H}_{\mathrm{E}}$ are symmetric in regards to the $Y$ axis and are indicated by dashed lines on the graphs. tion of the $\mathrm{F}$ layer. ${ }^{1,8} \mathrm{H}_{\mathrm{FC}}$ was applied along the longitudinal direction of the dots, as indicated in Fig. 1(b). The $\mathrm{T}_{\mathrm{B}}$ distributions were then determined in the range of 4 to $450 \mathrm{~K}$ from magnetic hysteresis loops measured by SQUID. All the loops were performed at the same reference $\mathrm{T}$ of $4 \mathrm{~K}$, following a specific FC procedure after Ref. 14. Typical hysteresis loops measured during the procedure are shown in Figs. 2(a) and 2(b). (i) The first step of the procedure consists in an initial FC from 450 to $4 \mathrm{~K}$ under positive saturating field. All the $\mathrm{AF}$ entities contributing to $\mathrm{EB}$ are then oriented toward this positive direction. After measurement of the hysteresis loop at $4 \mathrm{~K}$, one gets loop shift with the maximum amplitude $\left(\mathrm{H}_{\mathrm{E}, \min }=-\mathrm{H}_{\mathrm{E} \text {,max }}\right)$. (ii) The procedure then consists of gradually orienting AF entities toward the opposite direction. This is done by raising the $\mathrm{T}$ up to an intermediate annealing temperature $\left(\mathrm{T}_{\mathrm{a}}\right)$ and then by $\mathrm{FC}$ down to $4 \mathrm{~K}$ under a negative magnetic field. A hysteresis loop is measured at $4 \mathrm{~K}$ after this step. The above step is repeated for increasing $\mathrm{T}_{\mathrm{a}}$. At each increment, all the AF entities with $T_{B}$ comprised between $T_{a}$ and $4 \mathrm{~K}$ have been reoriented toward the negative direction. As seen in Fig. 2 we thus observe a gradual change in the amplitude and sign of $\mathrm{H}_{\mathrm{E}}$ when $\mathrm{T}_{\mathrm{a}}$ is increased since then more and more $\mathrm{AF}$ entities are reoriented toward the negative direction. Then by plotting $\mathrm{H}_{\mathrm{E}}$ as a function of $\mathrm{T}_{\mathrm{a}}$ [Figs. 2(b) and 2(c)], we get the integrand of the $T_{B}$ distribution. Indeed, $\mathrm{H}_{\mathrm{E}}$ at each increment of $\mathrm{T}_{\mathrm{a}}$ is proportional to the difference between the amount of entities oriented positively and negatively. ${ }^{8,14} \mathrm{We}$ emphasize that the purpose of using a fixed $\mathrm{T}$ for the measurement within this specific protocol is to get rid of spurious effects associated with the thermal variations in the physical parameters (AF anisotropy, $\mathrm{F}$ magnetization, and AF/F interfacial coupling strength) and thereby to derive more intrinsic information on the distributions of EB characteristics. This is not directly possible from measurements of $\mathrm{H}_{\mathrm{E}}$ at various $\mathrm{T}$.

Dependences of $\mathrm{H}_{\mathrm{E}}$ on $\mathrm{T}_{\mathrm{a}}$ for the continuous film and corresponding array of dots are plotted in Figs. 2(c) and 2(d), along with the values of $\mathrm{H}_{\mathrm{E} \text {,min }}$ and $\mathrm{H}_{\mathrm{E} \text {, max }}$. In both cases, we see the expected change in the amplitude and sign of $\mathrm{H}_{\mathrm{E}}$.
Both loops show two inflection points separated by a plateau, which then translates into bimodal distributions of $\mathrm{T}_{\mathrm{B}}$ determined from the derivative of $\mathrm{H}_{\mathrm{E}}$ versus $\mathrm{T}_{\mathrm{a}}{ }^{8}$ The high-T inflection point in the $\mathrm{H}_{\mathrm{E}}$ versus $\mathrm{T}_{\mathrm{a}}$ dependences is commonly observed. It is ascribed to AF grains, which undergo thermally activated magnetic reversal. ${ }^{6,8-10}$ It is also established that this high-T inflection point shifts toward lower $\mathrm{T}$ in the case of nanodots with respect to continuous films, as due to the reduction in the volume of the grains located at the dot edges. Indeed, these smaller grains become more prone to $\mathrm{T}$ activation as evidenced and discussed in earlier works. ${ }^{4,5,7}$ Our main point of interest here is the less studied low- $\mathrm{T}$ inflection point in the $\mathrm{H}_{\mathrm{E}}$ versus $\mathrm{T}_{\mathrm{a}}$ dependence, which is directly related to the low-T part of the $\mathrm{T}_{\mathrm{B}}$ distribution. For continuous films, we have recently related this low- $\mathrm{T}$ part of the $\mathrm{T}_{\mathrm{B}}$ distribution to disordered interfacial spins, ${ }^{8,11}$ which exhibit spin-glasslike behavior. $^{12,13}$ They are randomly distributed over the interface. They yield an effective distribution of coupling, $\mathrm{J}_{\mathrm{F} / \mathrm{AF}}$ across the F/AF interface. From Figs. 2(c) and 2(d), one can observe that for both the continuous film and the nanodots, the low- $\mathrm{T}$ part of the $\mathrm{T}_{\mathrm{B}}$ distributions extend up to around $200 \mathrm{~K}$ Indeed, above this $\mathrm{T}$ the $\mathrm{H}_{\mathrm{E}}$ versus $\mathrm{T}_{\mathrm{a}}$ variation levels off. Yet these low-T distributions yield differences in the relative amplitude of variations in $\mathrm{H}_{\mathrm{E}}$ after reversed $\mathrm{FC}$ between $\mathrm{T}_{\mathrm{a}}$ and $4 \mathrm{~K}$. At $200 \mathrm{~K}$, for the continuous film, $\mathrm{H}_{\mathrm{E}}$ is almost zero, meaning that $50 \%$ of the total amplitude of variation in $\mathrm{H}_{\mathrm{E}}$ has been completed; half of the $\mathrm{AF}$ entities are pointing in the initial positive $\mathrm{FC}$ direction and the other half in the negative $\mathrm{FC}$ direction. It is equivalent to say that $50 \%$ of the AF entities contribute to the low-T part of the $\mathrm{T}_{\mathrm{B}}$ distribution for continuous films which implies that the weight of the contribution to $\mathrm{EB}$ of interfacial spin glasslike regions (low-T) equals that of $\mathrm{AF}$ grains (high-T). The amount of reversed entities at $\mathrm{T}_{\mathrm{a}}$ can be calculated from the normalized ratio $\left(\left|\mathrm{H}_{\mathrm{E}}\right|-\left|\mathrm{H}_{\mathrm{E}, \min }\right|\right) /\left(\mathrm{H}_{\mathrm{E}, \max }-\left|\mathrm{H}_{\mathrm{E}, \min }\right|\right)$ and is indicated on the right hand side scales of the graphs in Fig. 2. In contrast, for the dots at $200 \mathrm{~K}$, not only $50 \%$ but $75 \%$ of the total amplitude variation in $\mathrm{H}_{\mathrm{E}}$ has been completed. This 
(a)

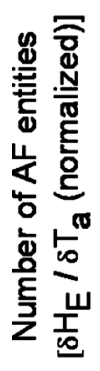

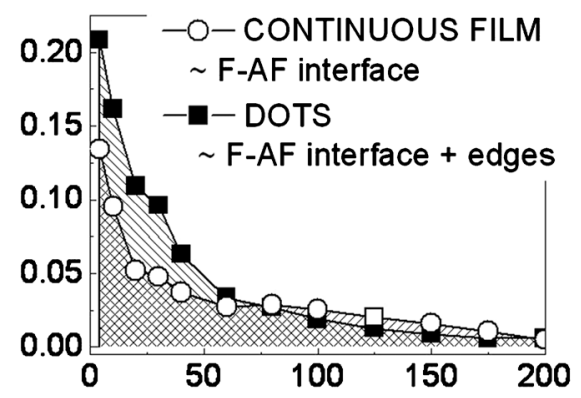

(b)

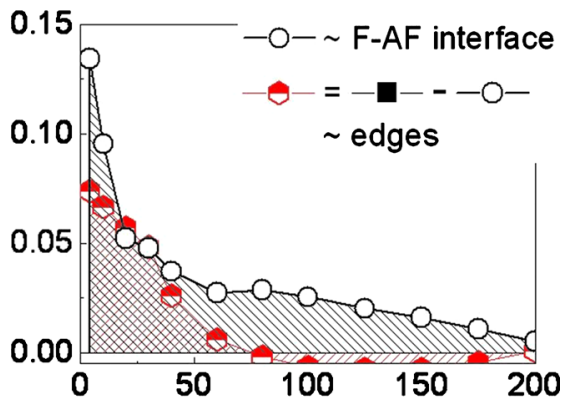

Blocking temperature $(\mathrm{K})$

$\left[\mathrm{T}_{\mathrm{a}}(\mathrm{K})\right]$

FIG. 3. (Color online) (a) Dependence on $\mathrm{T}_{\mathrm{a}}$ of the derivative $\delta \mathrm{H}_{\mathrm{E}} / \delta \mathrm{T}_{\mathrm{a}}$ as deduced from the full lines in Figs. 2(c) and 2(d), both for a continuous film and an array of $180 \times 100 \mathrm{~nm}^{2}$ dots. $\delta \mathrm{H}_{\mathrm{E}} / \delta \mathrm{T}_{\mathrm{a}} \mathrm{vs} \mathrm{T}_{\mathrm{a}}$ represent the blocking temperature distributions. The graphs are zoomed on the low $\mathrm{T}$ distributions. The normalization is dictated by the experimental results of Figs. 2(c) and 2(d): the integral of the distribution from 4 to $200 \mathrm{~K}$ equals $50 \%$ for the continuous film and $75 \%$ for the dots. (b) Difference between the $\mathrm{T}_{\mathrm{B}}$ distributions for the continuous film and for the dots as plotted from (a). This corresponds to the additional contribution from the edges of the dots. For comparison the $\mathrm{T}_{\mathrm{B}}$ distribution for the continuous film, which corresponds to the contribution of the $\mathrm{F}-\mathrm{AF}$ interface, is also reminded in (b).

means that the contribution to EB coming from the bulk of the AF layer (i.e., the high- $\mathrm{T}$ part of the $\mathrm{T}_{\mathrm{B}}$ distribution) represents only $25 \%$ of all magnetic entities influencing $\mathrm{EB}$ in nanodots.

In Fig. 3(a) the distributions of $T_{B}$ are plotted from the derivative of the loops of Figs. 2(c) and 2(d). A zoom of the low-T distribution, which is the point of interest here, allows us to clearly see the additional contribution for the dots, which we suspected from the interpretation of Figs. 2(c) and 2(d). We believe that this additional contribution is due to AF entities located at the edges of the dots. In these regions, due to the physical patterning, there is a break in the lateral coherence within the $\mathrm{AF}$ in addition to grains cutting and edges roughness. ${ }^{4,5} 7$ This may lead to additional localized spin disorder at the F/AF interface in the vicinity of the nanodot edges. Thus, patterning creates edge defects, which work as additional location for the formation of spin glasslike regions. The latter add to the randomly distributed spin glasslike regions present in both continuous films and dots and which are due to the random distribution of frustrated areas over the F/AF interface. ${ }^{8,12,13}$ An idea of the distribution of $\mathrm{T}_{\mathrm{B}}$ brought by the edge contribution may thus be visualized by plotting the difference between the low-T part of the $T_{B}$ distributions measured for the dots and for the continuous film. This derived edge contribution is plotted along with the interfacial contribution in Fig. 3(b). From this figure, one can say that the relative contribution of edge AF entities is not negligible in regards to that of interfacial AF entities. Indeed for our $180 \times 100 \mathrm{~nm}^{2}$ dots, and approximating the grain size to a reasonable value of $20 \mathrm{~nm},{ }^{6}$ one gets about 30 grains affected by the edges to be compared to a total of 50 grains affected by the interface. One cannot however quantitatively compare the relative number of entities with a bare calculation of the ratio between interface and edge of the dots since spin-glasslike regions do not cover the whole interface and edges. From our understanding, we can however already qualitatively predict that this contribution of edge $\mathrm{AF}$ entities should depend linearly with the dots edge length along the F/AF interface when varying the dots size.

In conclusion, for F/AF bilayers, we evidenced the twofold consequences of patterning. It not only alters the grain size distribution but it also provides additional locations for the formation of F/AF spin-glasslike regions with low $T_{B}$. Both effects weaken the thermal stability of exchange bias after patterning. Further reducing the dot sizes will increase the above effects. The preservation of $\mathrm{H}_{\mathrm{E}}$ and $\mathrm{T}_{\mathrm{B}}$ above working $\mathrm{T}$ for deeply submicrometric dots, which is needed for the implementation of devices, will require to address these issues related to edge effects. If overcoming the alteration of the grain size distribution due to patterning may be attempted by increasing the coupling between $\mathrm{AF}$ grains or working with smaller grains of higher anisotropy, limiting the formation of spin-glasslike regions at the F/AF interface in the vicinity of the edges may be challenging.

Financial support from the Rhône-Alpes region via the project Cluster MICRO-NANO STARAC is acknowledged.

${ }^{1}$ B. Dieny, V. S. Speriosu, S. S. P. Parkin, B. A. Gurney, D. R. Wilhoit, and D. Mauri, Phys. Rev. B 43, 1297 (1991).

${ }^{2}$ J. Nogués and I. K. Schuller, J. Magn. Magn. Mater. 192, 203 (1999); A. E. Berkowitz and K. Takano, ibid. 200, 552 (1999); R. L. Stamps, J. Phys. D: Appl. Phys. 33, R247 (2000); M. Kiwi, J. Magn. Magn. Mater. 234, 584 (2001).

${ }^{3}$ J. Hérault, R. C. Sousa, C. Ducruet, B. Dieny, Y. Conraux, C. Portemont, K. Mackay, I. L. Prejbeanu, B. Delaet, M. C. Cyrille, and O. Redon, J. Appl. Phys. 106, 014505 (2009).

${ }^{4}$ V. Baltz, J. Sort, B. Rodmacq, B. Dieny, and S. Landis, Phys. Rev. B 72, 104419 (2005).

${ }^{5}$ G. Vallejo-Fernandez and J. N. Chapmam, Appl. Phys. Lett. 94, 262508 (2009).

${ }^{6}$ K. O'Grady, L. E. Fernandez-Outon, and G. Vallejo-Fernandez, J. Magn. Magn. Mater. 322, 883 (2010).

${ }^{7}$ V. Baltz, J. Sort, S. Landis, B. Rodmacq, and B. Dieny, Phys. Rev. Lett. 94, 117201 (2005).

${ }^{8}$ V. Baltz, B. Rodmacq, A. Zarefy, L. Lechevallier, and B. Dieny, Phys. Rev. B 81, 052404 (2010).

${ }^{9}$ E. Fulcomer and S. H. Charap, J. Appl. Phys. 43, 4190 (1972).

${ }^{10}$ M. D. Stiles and R. D. McMichael, Phys. Rev. B 60, 12950 (1999).

${ }^{11}$ K. Takano, R. H. Kodama, A. E. Berkowitz, W. Cao, and G. Thomas, Phys. Rev. Lett. 79, 1130 (1997).

${ }^{12}$ A. P. Malozemoff, Phys. Rev. B 35, 3679 (1987).

${ }^{13}$ M. Ali, P. Adie, C. H. Marrows, D. Greig, B. J. Hickey, and R. L. Stamps, Nature Mater. 6, 70 (2007).

${ }^{14}$ S. Soeya, T. Imagawa, S. Mitsuoka, and S. Narishige, J. Appl. Phys. 76, 5356 (1994). 